\title{
el Consejo Estatal de Población de San Luis Potosí: APUNTES PARA SU HISTORIA*
}

\author{
José Alberto Juárez Miranda**
}

\section{Resumen}

Basado en un aspecto de la descripción documental -la historia institucional- se ofrece una breve reseña de la historia del Consejo Estatal de Población de San Luis Potosí (Coesposlp). Se abordan los antecedentes a nivel nacional y local, funciones y nombre de sus titulares.

Palabras clave: población, historia, San Luis Potosí.

\begin{abstract}
Based on a documental description aspect -institutional history-, this article offers a brief overview about State Council of the Population of San Luis Potosi's history; besides, it presents background at national and local levels, institution functions and name of its directors.
\end{abstract}

Keywords: population, history, San Luis Potosí.

* Versión ampliada de una conferencia dictada el 31 de octubre de 2013 en la capilla de Aránzazu del Museo Regional Potosino, en la ciudad de San Luis Potosí, S. L. P., con motivo de la celebración del xxv aniversario de la creación del Consejo Estatal de Población de San Luis Potosí.

** Archivo General del Estado de San Luis Potosí. 
Este texto es parte de los resultados del proceso de gestión documental ${ }^{1}$ que se lleva a cabo en el Archivo General del Estado de San Luis Potosí (AGESLP), específicamente en lo que se refiere a la descripción en el nivel de fondo, donde, según las normas vigentes, uno de los elementos a contemplar es la historia de la institución productora de los documentos. Como norma para la descripción documental, el AGESLP emplea la segunda edición de la Norma Internacional General de Descripción Archivística, por sus siglas en inglés, ISAD $(\mathrm{G}),{ }^{2}$ la cual propone 26 elementos que pueden aplicarse desde el nivel más general -fondo documental- ${ }^{3}$ hasta el más específico -la unidad documental simple o documento- ${ }^{4}$. Dichos elementos están agrupados en siete áreas de información descriptiva: identificación -información esencial para identificar la unidad de descripción-, contexto -información sobre el origen y custodia de la unidad de descripción-, contenido y estructura -información relativa al objeto y organización de la unidad de descripcióncondiciones de acceso y utilización -información relativa a la accesibilidad de la unidad de descripción- documentación asociada -información relativa a aquellos documentos que tienen relación significativa con la unidad de descripción- notas -información especial que no ha podido incluirse en las demás áreas- y control de la descripción -información relativa al cómo, cuándo y quién ha elaborado la descripción archivística.

La historia institucional forma parte del área 2 y, de acuerdo con la ISAD (G), el objetivo de la inclusión de este elemento es proporcionar la historia del productor o los productores de la unidad de descripción para situar la documentación en su contexto y hacerla más comprensible; para tal efecto,

1 La gestión documental se define como el conjunto de normas, técnicas y conocimientos aplicados al tratamiento de los documentos desde su diseño hasta su conservación permanente. También puede entenderse como sinónimo de administración de documentos: "conjunto de métodos y prácticas destinados a planear, dirigir y controlar la producción, circulación, conservación, uso, selección y disposición final de los documentos de archivo, con el propósito de lograr la eficiencia y eficacia en el manejo de los mismos a lo largo de su ciclo de vida". Cruz Mundet, Administración de documentos y archivos, p. 18.

2 Consejo Internacional de Archivos, (CIA), ISAD (G).

3 Se entiende por fondo documental el "conjunto de documentos, con independencia de su tipo documental o soporte, producidos orgánicamente o acumulados y utilizados por una persona física, familia, o entidad en el transcurso de sus actividades y funciones como productor". Consejo Internacional de Archivos, ISAD (G), p. 17.

$4 \mathrm{El}$ concepto se define como "información que ha quedado registrada de alguna forma con independencia de su soporte o características". Consejo Internacional de Archivos, ISAD (G), p. 16. 
en el proceso de recopilación y redacción se sugiere considerar, en forma abreviada, datos significativos en torno al origen, evolución, desarrollo y trabajo de la entidad o entidades responsables de la producción de la unidad de descripción..$^{5}$ Los lineamientos específicos en torno a la identificación de los datos requeridos los proporciona una herramienta complementaria: la ISAAR (CPF), ${ }^{6}$ particularmente en la denominada Área de Descripción, en la que se dispone incluir fechas de existencia, historia, lugares, estatuto jurídico, funciones, ocupaciones, actividades, atribuciones (fuentes legales), estructura interna y contexto general.

De las fechas de existencia se propone incluir el establecimiento, fundación, legislación sobre sus competencias y disolución; sobre la historia, registrar en forma narrativa o cronológica los principales acontecimientos, actividades, logros y funciones; en torno a los lugares, precisar las distintas sedes, así como las fechas de permanencia; respecto del estatuto jurídico, la naturaleza y tipo de institución, así como las fechas de vigencia del estatuto; referente a las funciones, ocupaciones y actividades, sugiere anotar la naturaleza y periodo de realización; en lo concerniente a las atribuciones (fuentes legales), recomienda basarse en el estudio del marco normativo aplicable para ubicar el origen de sus competencias legales, funciones, responsabilidades, jurisdicción y periodo de vigencia. ${ }^{7}$ En la medida en que las fuentes documentales lo permiten, este trabajo intenta reflejar la aplicación de la metodología propuesta por las normas indicadas, particularmente al proceso de construcción de la historia institucional en el nivel de fondo, del fondo documental Consejo Estatal del Población de San Luis Potosí (Coesposlp), el cual forma parte del acervo que custodia el Archivo General del Estado de San Luis Potosí (Ageslp).

Las fuentes documentales que nutren y dan sustento al contenido de esta breve reseña son de orden jurídico y administrativo: acuerdos y convenios celebrados entre el gobierno del estado de San Luis Potosí y la Secretaría de Gobernación (Segob), la Ley General de Población, el Acuerdo de Creación del Coesposlp, su reglamento interno y un expediente de carácter

5 Consejo Internacional de Archivos, ISAD (G), p. 27.

6 Consejo Internacional de Archivos, IASAR (CPF).

7 Consejo Internacional de Archivos, ISAAR (CPF), pp. 20-27. 
administrativo en el que se contienen los antecedentes legales hasta ahora desconocidos.

La historia de esta institución se revela en tres etapas perfectamente identificadas, pero al mismo tiempo entrelazadas.

\section{Primera etapa (1947 a 1983)}

Las actividades en las que posteriormente participaría el Coesposlp se encuentran, durante este periodo, estrechamente ligadas a la Segob y al Consejo Nacional de Población (Conapo).

En 1947, a unos cuantos años de la culminación de la Segunda Guerra Mundial, el Gobierno de la República Mexicana promulgó la primera versión de la Ley Nacional de Población. Por medio de este documento el Estado mexicano manifiesta su interés por resolver los problemas demográficos del país, particularmente el aumento de la población, su distribución racional, la fusión étnica de grupos, la asimilación de extranjeros al medio nacional, la protección de los connacionales en sus actividades económicas, profesionales, artísticas e intelectuales y la incorporación y mejora de vida de los grupos indígenas. Todo lo cual se pretendió llevar a cabo a través de la Segob. En el artículo $3^{\circ}$ de dicha Ley se perfila la competencia que los gobiernos de los estados de la federación tendrían en la aplicación y ejecución de cada uno de los fines de la política demográfica nacional-competencia, en realidad, limitada, pues al mismo tiempo se enfatiza que la definición de normas, iniciativas de conjunto y la coordinación de labores serían tareas exclusivas de la Segob. ${ }^{8}$

Casi treinta años después la referida Ley es abrogada y se expide otra, mediante la cual se crea el Conapo. ${ }^{9}$ En esta nueva Ley se refuerza la injerencia de los estados en la materia. En el capítulo IV, referente a la emigración, el artículo 80 bis -de las reformas-, establece que el Gobierno federal, en coordinación con los gobiernos de las entidades federativas y municipales, deberá:

8 Ley General de Población, Diario Oficial de la Federación (en adelante, DOF), 27 de diciembre de 1947.

9 Ley General de Población, DOF, 7 de enero de 1974. 
I. Promover el desarrollo y fomentar el arraigo de los mexicanos al territorio nacional;

II. Crear programas para atender los impactos de la emigración en las comunidades de origen, especialmente en lo relacionado con la problemática de la desintegración familiar y con la atención de personas en situación de vulnerabilidad.

En el capítulo VI, referente al Registro de Población e Identidad Nacional, el artículo 90 señala la participación -en calidad de auxiliares-, de las autoridades estatales en el Registro de Población e Identificación Personal y en todas las actividades del consejo; el artículo 93 responsabiliza a las autoridades locales para que contribuyan a la integración del Registro Nacional de Población facultando para tal efecto a la Segob para celebrar con ellas convenios cuyos propósitos sean adoptar la normatividad al respecto; recabar la información relativa a los nacimientos y defunciones de las personas, a fin de integrar y mantener permanentemente actualizado el Registro Nacional de Población y, finalmente, incluir en el acta de nacimiento correspondiente la Clave Única de Registro de Población (CURP). El artículo 94 de las reformas insiste, asimismo, en que las autoridades de los estados y municipios serán auxiliares de la Segob en las funciones relativas al registro de población.

En cumplimiento de lo estipulado en la Ley General de Población sobre la responsabilidad de los estados en la aplicación de la política nacional de población, el Gobierno del estado de San Luis Potosí y el Coesposlp han venido celebrando, de manera conjunta, diversos acuerdos de coordinación con la Segob y el Conapo. Es posible que el primero de éstos se haya firmado en 1983, ${ }^{10}$ durante el último tercio de la gestión del profesor Carlos Jonguitud Barrios, gobernador constitucional del estado de San Luis Potosí. ${ }^{11}$ Lo que

10 "Acuerdo de Coordinación que celebran por una parte, el Ejecutivo Federal, a través de la Secretaría de Gobernación con la participación del Consejo Nacional de Población, y por la otra, el Gobierno del Estado de San Luis Potosí”, diciembre de 1983. Se cita en el Decreto de 1988, referente a la creación de Coesposlp, pero hasta ahora no ha sido posible localizar su publicación.

11 Jonguitud gobernó el estado de San Luis Potosí del 29 de septiembre de 1979 al 25 de septiembre de 1985. Montejano, San Luis Potosí, p. 184. 
sí consta son varias ratificaciones al referido acuerdo: $1990,{ }^{12} 1993,{ }^{13} 1994,{ }^{14}$ $2003,{ }^{15}$ y $2010 .{ }^{16}$ En congruencia con la responsabilidad estatal establecida en la Ley, el contenido sustantivo de todos ellos se ha enfocado tanto en definir las bases de cooperación como en establecer, coordinar y evaluar procedimientos y acciones concretas en la materia para el territorio local.

\section{Segunda etapa (1985 a 1988)}

En este periodo se encuentran los orígenes legales de la creación del Coesposlp. No obstante, ante la carencia de una investigación documental, este hecho era totalmente desconocido hasta el momento previo a esta investigación.

Al final de la administración del profesor y licenciado Carlos Jonguitud Barrios, se firmó un Acuerdo Gubernamental para la Constitución del Consejo Estatal de Población. Entre sus signatarios figuró el propio gobernador, además del profesor J. Refugio Araujo del Ángel, secretario general de Gobierno, y el licenciado Gerónimo Martínez García, director general del Conapo, quien además fungió como testigo de honor. El artículo primero transitorio del acuerdo estipula que el mismo entraría en vigor al día siguiente de su firma, ocurrida el primero de agosto de $1985 .{ }^{17} \mathrm{El}$ artículo $2^{\circ}$ transitorio informa que el documento en cuestión sería publicado en el Periódico Oficial del Estado; aunque no se ha verificado tal publicación, ello no debe interpretarse como sinónimo de invalidez, pues como se estipula en el documento, el hecho de haber sido firmado lo autentifica y le da vigencia.

El día de la firma del acuerdo fue instalado el consejo; para tal efecto, el Comité de Planeación para el Desarrollo del Estado (Coplade) convocó a una ceremonia oficial que se celebraría a las once horas en el Salón de Gobernadores de Palacio de Gobierno. A ella asistieron el Arquitecto

12 Ratificación del "Acuerdo de Coordinación”, DOF, 3 de mayo de 1990.

13 "Acuerdo de Coordinación", DOF, 22 de diciembre de 1993.

14 “Acuerdo de Coordinación", Periódico Oficial del Gobierno del Estado Libre y Soberano de San Luis Potosí, 8 de febrero de 1994.

15 "Acuerdo de Coordinación", POESLP, 26 de abril de 2003.

16 “Acuerdo de Coordinación”, POESLP, 30 de noviembre de 2010.

17 "Acuerdo gubernamental para la constitución del Consejo Estatal de Población - propuesta-, $1^{\circ}$ de agosto de 1985", AGESLP, Fondo Archivo General del Estado de San Luis Potosí (en adelante F. AGESLP), Exp. 570/0/20, ff. 12-21. 
Benito Delgadillo Amaro, secretario de Programación y Presupuesto Estatal y coordinador general del Coplade; el profesor J. Refugio Araujo del Ángel, secretario general de Gobierno, quien presentó el acuerdo; y Carlos Jonguitud Barrios, gobernador del estado, quien, merced a sus atribuciones de máximo jerarca del gobierno potosino, tomó protesta a los integrantes de la naciente institución; e igual que el licenciado Gerónimo Martínez García, director general del Conapo, dirigió unas palabras a los asistentes. ${ }^{18}$

En esta etapa de su historia, conforme con el artículo $2^{\circ}$ del citado acuerdo, el Coesposlp tuvo como objetivo elaborar y promover la ejecución de acciones y programas específicos a fin de que la dinámica y distribución de la población en el territorio estatal fueran acordes con el Plan San Luis; ${ }^{19}$ asimismo, buscó la promoción de programas de población y desarrollo socioeconómico. Para tal efecto se le asignaron las siguientes funciones:

I. Elaborar, en coordinación con el Conapo, el Programa Estatal de Población a fin de promover la incorporación de la población a los planes de desarrollo socioeconómico del estado y vincular los objetivos de éste con las necesidades que plantean los fenómenos demográficos a nivel local, regional y nacional.

II. Fungir como coordinador de las dependencias de los gobiernos estatal y federal que tengan funciones relacionadas con la materia demográfica; así como de la participación de los municipios y de los sectores social y privado en la ejecución del Programa Estatal de Población.

III. Coordinar la ejecución de las acciones previstas en el Programa Estatal de Población, procurando la congruencia y complementación con el Programa Nacional de Población.

18 "Reunión de trabajo del Comité de Planeación para el Desarrollo del Estado para la constitución del Consejo Estatal de Población", $1^{\circ}$ de agosto de 1985. AgESLP. F. AGeSLP, exp. $570 / 0 / 20$ ff. 24 .

19 Así fue denominado el Programa Estatal de Desarrollo implementado por el Gobierno del estado de San Luis Potosí en el periodo 1985-1991. "Plan San Luis, 1985-1991", POESLP, 25 de marzo de 1986. 
IV. Validar la información demográfica en el estado y promover la generación de aquella que sirva de base para el conocimiento y la toma de decisiones.

v. Evaluar, junto con el Conapo, los programas realizados, a fin de asegurar el éxito de la instrumentación de la política nacional de población.

VI. Integrar un banco de información sociodemográfica que sirva para ampliar el conocimiento acerca de los fenómenos demográficos y los principales problemas que enfrenta la población.

VII. Promover, apoyar y coordinar estudios que se efectúen para mejorar permanentemente la programación estatal de la población y del desarrollo.

VIII. Evaluar los programas que lleven a cabo las diferentes dependencias u organismos del sector público y se hayan formulado de acuerdo con el Programa Estatal de Población, y proponer las medidas pertinentes para su cumplimiento.

Respecto al gobierno y estructura interna del Coesposlp, el artículo $4^{\circ}$ informa que éste se integraría por:

I. Un presidente, que corresponderá, al C. Gobernador del estado, el cual tendrá voto de calidad.

II. Por los delegados o representantes en el estado de las dependencias federales siguientes: Secretaría de Programación y Presupuesto, Secretaría de Salud, Secretaría de Educación Pública, Secretaría de Desarrollo Urbano y Ecología, Secretaría de Trabajo y Previsión Social, Secretaría de Agricultura y Recursos Hidráulicos, Secretaría de Comunicaciones y Transportes, Secretaría de Turismo, Instituto Mexicano del Seguro Social e Instituto de Seguridad y Servicios Sociales para los Trabajadores del Estado.

III. Por la Secretaría General de Gobierno, Secretaría de Programación y Presupuesto Estatal, Secretaría de Desarrollo Urbano y Ecología Estatal, Secretaría de Fomento Económico, Secretaría de Comunicaciones y Transportes Estatal, Centro Estatal de Estudios Municipales e Instituto Estatal de la Vivienda. 
IV. Por los organismos de los sectores social y privado que se vinculen con el fenómeno poblacional del estado.

v. Por un secretario técnico, que estará coordinado por un Secretario Técnico nombrado por el Gobernador del Estado.

VI. Un equipo técnico, que tendrá a su cargo la formulación de estudios y programas en materia de población, el cual contará con los recursos humanos, materiales y financieros que determine el Consejo. ${ }^{20}$

Una vez instalado el Coesposlp, era necesario dar a conocer su naturaleza e importancia al interior y exterior del mismo. Por tal motivo, el 29 de agosto de 1985 se celebró un ciclo de conferencias dirigidas a los servidores públicos que lo integraron. El evento fue organizado por el arquitecto Delgadillo, quien en la ceremonia de firma del acuerdo había sido designado como secretario técnico del Consejo Estatal de Población. ${ }^{21}$

Entre 1980 y 1990 la población aumentó considerablemente a lo largo y ancho del territorio nacional; en la entidad potosina llegó a casi medio millón de habitantes, lo que significaba un incremento de $40 \%$ con respecto a la década de $1970 .{ }^{22}$ Sin duda, tal estadística motivó a las autoridades en su decisión de instalar el Coesposlp. Entre agosto de 1985 y abril de 1988 esta institución estuvo vigente y realizó en forma sistemática y continua las atribuciones que le fueron encomendadas en el acuerdo que le dio origen; además participó en distintos eventos celebrados en territorio potosino y más allá de sus fronteras. Lo primero lo confirma el licenciado Fernando Mercado Nordhausen, secretario técnico del Consejo Estatal de Población, pues el 23 de octubre de 1987, mediante oficio, agradece al licenciado Adalberto Noyola Vázquez, secretario general de Gobierno, su asistencia a la ceremonia de instalación del consejo; de lo segundo da cuenta una nota fechada el 21 de abril de 1988, la cual informa de la participación de

20 "Acuerdo gubernamental para la constitución del Consejo Estatal de Población -propuesta-, $1^{\circ}$ de agosto de 1985". AGESLP. F. AGESLP, exp. 570/0/20, ff. 14-17.

21 Arquitecto Benito Delgadillo Amaro, secretario de Programación y Presupuesto Estatal y Secretario Técnico del Consejo Estatal de Población, al C. Prof. J. Refugio Araujo del Ángel. Oficio por medio del cual le gira invitación al ciclo de conferencias dirigidas a los servidores públicos que integran el Consejo Estatal de Población, 23 de agosto de 1985. Se anexa programa de conferencias, ibid., ff. 28 y 29.

22 Instituto Nacional de Estadística y Geografía (Inegi) Estadísticas históricas de México, p. 37. 
Nordhausen en la Reunión Estatal del Subcomité de Descentralización de la Industria, el Comercio Exterior y el Abasto. ${ }^{23}$

\section{Tercera etapa (1988-)}

Si se toma como punto de referencia la publicación, en 1988, del Acuerdo de Creación - del cual se celebró, en 2013, su Xxv aniversario-, el origen oficial del Coesposlp se enmarca en el contexto histórico de las dos últimas décadas del siglo xx, particularmente el periodo de 1983 a 1996, cuando se crean los consejos estatales de población de las 32 entidades federativas de la República mexicana. El pionero fue consejo del estado de Morelos, fundado el 30 de diciembre de 1983; mientras que el último fue el del Distrito Federal, creado el 12 de abril de 1996. Trazado el camino, en 1984 se fundaron 22 consejos; en 1986, uno más; en 1987, tres; en 1988, dos; en 1989, 1992 y 1996, uno.

El Coesposlp fue creado en 1988 y ocupa el lugar número veintiocho en el orden cronológico. ${ }^{24}$ Se fundó durante el gobierno provisional de Leopoldino Ortiz Santos, quien al considerar la necesidad de formular acciones y programas específicos de trabajo en el estado, y en congruencia con la política nacional de población, dictó el 2 de septiembre un acuerdo administrativo, publicado el 14 de octubre de $1988 .{ }^{25}$ Esta nueva institución nació como un organismo público, descentralizado, con personalidad jurídica y patrimonio propios, teniendo la capital del estado como sede para sus instalaciones. En esta etapa de su historia, según el artículo $4^{\circ}$ del citado acuerdo, el consejo tuvo como objetivos integrar a la población potosina, desarrollar el proceso de planeación demográfica estatal y local y ejecutar acciones específicas en materia de población, con el fin de que la dinámica y distribución de la entidad fueran adecuadas con los programas de desarrollo socioeconómico.

23 El licenciado Fernando Mercado Nordhausen, secretario técnico del Consejo Estatal de Población, al licenciado Adalberto Noyola Vázquez, secretario general de Gobierno. Oficio por medio del cual le agradece su asistencia a la ceremonia de instalación del consejo, 23 de octubre de 1987. AGESLP. F. AGESLP, exp. 570/0/20.

24 Secretaría de Gobernación. Consejo Nacional de Población. México: Segob-Conapo, 2013. En línea: http//www.conapo.gob.mx. Consultado el 6 de septiembre de 2013.

25 "Acuerdo administrativo. Se crea el Consejo Estatal de Población de San Luis Potosí", Periódico Oficial del Gobierno del Estado de San Luis Potosí (POEsLP), 14 de octubre de 1988. Cabe observar que el contenido de este acuerdo en esencia resulta idéntico al de su antecesor, firmado el 5 de agosto de 1985. 
En 1994, siendo gobernador del estado el licenciado Horacio Sánchez Unzueta, y titular del Coesposlp el licenciado Leonardo Hernández Covarrubias, el Gobierno del estado de San Luis Potosí y la Segob firmaron un nuevo acuerdo de coordinación que actualizó los firmados en 1990 y $1993 .{ }^{26}$

Diez años después de la creación del consejo, el licenciado Fernando Silva Nieto, gobernador constitucional del estado, expuso que las expectativas de su gobierno en materia de planeación y desarrollo económico y social registraban variantes que exigían una adecuación a las políticas de crecimiento demográfico de cara al Plan Estatal de Población; asimismo, que con base en las reformas a la Ley Orgánica de la Administración Pública del Estado de San Luis Potosí, resultaba imperativa la actualización del acuerdo por el que se creó el consejo. En consecuencia, dictó un decreto administrativo por medio del cual modificó y adicionó el susodicho acuerdo. Este documento fue publicado el 4 de febrero de $1998 .{ }^{27}$ Entre las modificaciones trascendentales se encuentran los objetivos, los cuales precisan que las acciones emprendidas por el consejo deberán enfocarse en la problemática regional. Se agrega que el patrimonio de la institución se conformará además por recursos financieros provenientes de organismos nacionales e internacionales.

En 2003, siendo gobernador del estado el licenciado Fernando Silva Nieto, y titular del Consejo Estatal de Población el ciudadano Fernando Díaz de León Cardona, el Gobierno del estado de San Luis Potosí y la Segob firmaron un nuevo acuerdo de coordinación que actualizó el firmado nueve años atrás. ${ }^{28}$

En ese mismo año se cubre por fin una necesidad pendiente en materia de reglamentación y estructura interna del Coesposlp: el 30 de julio se publica su Reglamento Interno. ${ }^{29}$ En su contenido se lee que el consejo quedaría integrado por un Presidente, el ciudadano gobernador del estado en turno; un secretario técnico, designado por el presidente del consejo; y los titulares de las siguientes dependencias y entidades: Secretaría General

26 “Acuerdo de Coordinación”, POESLP, 8 de febrero de 1994.

27 "Decreto Administrativo", POESLP, 4 de febrero de 1998.

28 "Acuerdo de Coordinación”, pOESLP, 26 de abril de 2003.

29 "Reglamento Interno del Consejo Estatal de Población”, POEslp, 30 de julio de 2003. 
de Gobierno, Secretaría de Desarrollo Social y Regional, Secretaría de Planeación del Desarrollo, Secretaría de Ecología y Gestión Ambiental, Secretaría de Educación de Gobierno del Estado, Secretaría de Finanzas, Secretaría de Desarrollo Agropecuario y Recursos Hidráulicos, Dirección de Servicios de Salud en el Estado, Secretaría de Desarrollo Económico, Oficialía Mayor, Desarrollo Integral de la Familia y, Coordinación General de Comunicación Social.

El Reglamento revela también la breve estructura diseñada para la dirección y operación del consejo: una Dirección General y una Dirección de Área de Coordinación Institucional. Además señala sus atribuciones principales: elaborar, ejecutar y evaluar el Programa Estatal de Población; participar en el seno del Comité de Planeación para el Desarrollo del Estado con el fin de promover e inducir la incorporación del criterio demográfico en los programas de desarrollo estatal y presidir el Subcomité Especial de Política Poblacional; apoyar la comunicación en población y desarrollar una base de datos sociodemográficos de la entidad; finalmente, proponer los mecanismos de concertación de acciones que permitan dar cumplimiento a los objetivos del Programa Estatal de Población, en coordinación con los del Programa Nacional.

En el umbral del siglo XxI, durante el gobierno del doctor Fernando Toranzo Fernández y siendo titular del Coesposlp la maestra María Teresa Galicia Saldaña, se publicaron dos importantes documentos: en 2010, un nuevo acuerdo de coordinación cuyo objetivo era ratificar el compromiso de las autoridades potosinas de compaginar y evaluar la aplicación de la política de población en la entidad; y en 2012, el decreto administrativo que reforma el acuerdo por el cual se creó el Consejo Estatal de Población. El objetivo de este documento es reforzar el correcto funcionamiento del consejo, y para tal efecto se adecua la estructura, se precisan las atribuciones del titular y se establece el órgano encargado de asesorar y realizar las recomendaciones necesarias en materia de política de población en el territorio potosino. ${ }^{31}$

30 “Acuerdo de Coordinación”, POESLP, 30 de noviembre de 2010.

31 "Decreto Administrativo que reforma el Acuerdo por el cual se crea el Consejo Estatal de Población”, POESLP, 27 de noviembre de 2012. 


\section{Actividades}

Sería prolijo enumerar la infinidad de importantes y muy variadas actividades que realiza el Coesposlp, ya sea en forma individual o coordinada con otras instancias de gobierno municipal, estatal, federal e internacional, además de asociaciones civiles, instituciones culturales, educativas y de investigación, a lo largo de sus ya casi treinta años de existencia. Sólo a manera de ejemplo, y en aras de la brevedad requerida, a continuación se indican algunas de las acciones de los últimos años.

1. Acciones de política de población a través de la cooperación para el desarrollo. Capacitación, conferencias, foros y exposiciones dirigidas a los migrantes de la casa de caridad Hogar del Migrante, con el objetivo de reflexionar, sensibilizarlos, prevenirlos de situaciones de riesgo y brindarles estrategias para el autocuidado. En el marco del Día Internacional de la Mujer, conferencias, exposiciones, jornadas, monólogos de enfoque académico y formativo con perspectiva de género. En actividades para conmemorar el Día del Niño y la Niña, cursos, talleres y exposiciones que promueven el autocuidado de la población infantil y difunden el conocimiento de los derechos sexuales y reproductivos. Como parte de las acciones para prevenir, atender, sancionar y erradicar la violencia hacia las mujeres, diversos eventos con perspectiva de género, difundiendo protocolos de atención a la violencia contra las mujeres y análisis de instrumentos de política pública.

En el Programa de Cultura Institucional con Perspectiva de Género, el cual comprende cuatro ejes de intervención -comunicación, trato, capacitación y desarrollo de habilidades-, pláticas, talleres, conferencias y dinámicas que fortalecen la igualdad y equidad de género en las áreas de trabajo. En la Semana Nacional de Ciencia y Tecnología, diversos talleres con temas como "prevención de la violencia de género", "masculinidad", "derechos sexuales y reproductivos" y "equidad de género". En el fortalecimiento de las capacidades interculturales sobre la prevención de la violencia de género en comunidades náhuatl y tenek, participación en la feria informativa y de servicios, promocionando información referente a las acciones y servicios que brinda la institución; así como programas que se están implementando para prevenir la violencia hacia las mujeres. 
2. Acciones que fortalecen la cultura demográfica en el estado. En el marco del Día Mundial del Medio Ambiente, la selección de dibujos ganadores del Concurso Nacional de Dibujo Infantil y Juvenil, el cual está dirigido a niños, niñas y jóvenes de entre 6 y 24 años. En temas de migración y derechos humanos, cursos de actualización, con el objetivo de que la sociedad civil, el personal de instituciones públicas y activistas fortalezcan el conocimiento y quehacer en defensa de los derechos de las personas en condición de migración. En torno a la conmemoración del Día Mundial de la Población, cuyo propósito es lograr un mundo donde cada persona joven alcance su pleno potencial, se promueven conferencias y foros. Con la finalidad de fortalecer la aplicación de la Política de Población en el estado, participación como enlace estatal en la firma de convenios de coordinación entre el Fondo de Población de las Naciones Unidas (UNFPA) y el Gobierno del estado de San Luis Potosí. ${ }^{32}$

Para el establecimiento del plan de trabajo del Programa Estatal de Cooperación (PEC), talleres de planeación, impartidos por representantes del UNFPA en colaboración con instituciones gubernamentales, organizaciones de la sociedad civil e instituciones académicas, con el objetivo de establecer estrategias de intervención que contribuyan a reducir la pobreza y las desigualdades socioeconómicas. Jornadas de actualización en derechos sexuales y reproductivos para su institucionalización como política de población, dirigidas a funcionarios públicos del Gobierno del estado de San Luis Potosí, organizaciones de la sociedad civil, academia y estudiantado.

Sobre los derechos humanos de los jóvenes, distintos eventos, en los que se plantean realidades y retos para la población adolescente y joven del país y el estado, con el propósito de construir la perspectiva de las juventudes y formular políticas públicas en su beneficio. En el Programa Prospera, participación como socio-expositor en la feria ;Vas! a Mover a México, donde se orienta e informa a jóvenes de diversas instituciones educativas para que continúen sus estudios hasta nivel profesional.

32 Consejo Estatal de Población, Informe anual de actividades, pp. 1-7. 


\section{Sede}

El Coesposlp ha tenido cuatro diferentes sedes a lo largo de su historia, todas localizadas en San Luis Potosí: entre 1983 y 1990, el Palacio de Gobierno del estado, ubicado en Jardín Hidalgo, zona centro; entre 1990 y 2000, la calle General Ignacio Zaragoza, en la misma zona; a principios del xxI, la calle Manuel del Conde; y desde 2010 a la fecha, la calle Miguel Barragán, número 515, en el barrio de San Miguelito.

\section{Titulares}

Desde su instalación en 1985 el Coesposlp ha tenido siete titulares nombrados, -una mujer y seis hombres-, entre quienes, por su formación académica, destacan un arquitecto, un abogado, dos licenciados en economía y una maestra en asuntos políticos. Todos con una amplia trayectoria en la administración pública, lo que en su momento les permitió, en mayor o menor grado, brindar su granito de arena en el proceso de construcción de la visión y misión actual de esta noble institución. A manera de homenaje me permitiré enunciar sus nombres y periodo de gestión.

1. Benito de Jesús Delgadillo Amaro. Arquitecto, catedrático, investigador, funcionario público en los gobiernos estatal y federal. En 1985 figura como secretario de Programación y Presupuesto Estatal, al mismo tiempo que secretario técnico del Coesposlp. ${ }^{33}$

2. Fernando Mercado Nordhausen. Funcionario público en el Gobierno estatal. En septiembre de 1987 fue nombrado director del Coesposlp, permaneciendo en el cargo hasta septiembre de $1991 .{ }^{34}$

3. Bruno Contreras Martel. Originario del municipio de Tancnahuitz, San Luis Potosí. Político y funcionario público de amplia trayectoria

33 Arquitecto Benito Delgadillo, secretario de Programación y Presupuesto Estatal y secretario Técnico del Consejo Estatal de Población, al profesor J. Refugio Araujo del Ángel, secretario general de Gobierno. Oficio por medio del cual hace invitación al ciclo de conferencias dirigidas a los servidores públicos que integran el Consejo Estatal de Población a celebrarse el 29 de agosto de 1985, 23 de agosto de 1985”. AGESLP. F. AGESLP, exp. 570/0/20, ff. 27.

34 Ageslp, Fondo Oficialía Mayor del Poder Ejecutivo del Estado. Sección Recursos Humanos (en adelante F. OM. RH). 
en su tierra natal y en los gobiernos estatal y federal. De 1989 a 1990 fue secretario técnico del Coesposlp. ${ }^{35}$

4. Leonardo Hernández Covarrubias. Economista y funcionario público en el Gobierno del estado. Entre 1990 y 1992 fue subdirector de Consejos Municipales en el Coesposlp; del 15 de enero de 1992 a marzo de 1993 se desempeñó como director, mientras que de marzo de 1993 a 1997, fungió como director general. ${ }^{36}$

5. Carlos González Orozco. Oriundo de la ciudad de San Luis Potosí. Político, economista y funcionario en la administración pública estatal y federal. De 1989 a 1997 ocupó diversos cargos en el Coesposlp: en 1989 y 1995 fue director; en 1992, subdirector, y en 1997, director general. $^{37}$

6. Fernando Díaz de León Cardona. Nació en la ciudad de San Luis Potosí. De amplia trayectoria periodística y en la administración pública estatal y federal. De 1992 hasta 2009 formó parte del equipo de trabajo del Coesposlp: entre 1992 y 1993 como capturista y de 1997 a 2009 como director general..$^{38}$

7. María Teresa Galicia Saldaña. Nació en la ciudad de San Luis Potosí. Funcionaria pública, maestra en asuntos políticos, catedrática, investigadora y consultora especializada. En septiembre de 2009 fue nombrada directora general del Coesposlp, cargo que desempeña hasta la fecha. ${ }^{39}$

Para concluir, manifiesto públicamente que esta información es sólo una breve y quizá incompleta perspectiva del devenir histórico de la institución en estudio. La precisión y detalles serán tarea de los actores de los acontecimientos aquí narrados, es decir, de los trabajadores y titulares. En todo caso, sirvan estos documentos y datos como punto de partida o complemento en el proceso de rescate y construcción de la verdad histórica de esta importante institución social, que ha demostrado, con sus ya casi

35 Idem.

36 Idem.

37 Idem.

38 Idem.

39 Idem. 
treinta años de existencia, ser de gran beneficio para los potosinos. Me permito recalcar la importancia de continuar la investigación y difundir sus resultados, porque sólo de esa manera se podrá tener una visión real, completa e incluyente, lo que no es un asunto menor, pues desde mi punto de vista, con esto se contribuye no sólo al conocimiento de la institución y a la construcción de la identidad y sentido de pertenencia de sus trabajadores, sino al conocimiento de la historia de las instituciones y de la administración pública del Estado de San Luis Potosí, al de la historia contemporánea de la región y, finalmente, al rescate y difusión del patrimonio documental y cultural de los habitantes de esta porción del territorio mexicano.

\section{Siglas}

AgeSLP. F. OM. RH, EP Archivo General del Estado de San Luis Potosí. Fondo Oficialía Mayor del Poder Ejecutivo del Estado. Sección Recursos Humanos.

AGESLP. F. AGESLP

DOF

POESLP
Archivo General del Estado de San Luis Potosí. Fondo Archivo General del Estado de San Luis Potosí.

Diario Oficial de la Federación.

Periódico Oficial del Gobierno del Estado Libre y Soberano de San Luis Potosí.

\section{Bibliografía}

"Acuerdo Administrativo. Se crea el Consejo Estatal de Población de San Luis Potosí" en Periódico Oficial del Gobierno del Estado Libre y Soberano de San Luis Potosí, 14 de octubre de 1988.

"Acuerdo de Coordinación celebrado entre el Ejecutivo federal y el Gobierno del estado con el objetivo de establecer, coordinar y evaluar la aplicación de la política de población en la entidad”, en Periódico Oficial del Gobierno del Estado Libre y Soberano de San Luis Potosí, 30 de noviembre de 2010. Edición extraordinaria. 
"Acuerdo de Coordinación que celebran el Ejecutivo Federal, a través de la Secretaría de Gobernación, con la participación del Consejo Nacional de Población y el Poder Ejecutivo del Estado de San Luis Potosí", en Diario Oficial de la Federación, 22 de diciembre de 1993.

"Acuerdo de Coordinación que celebran el Ejecutivo Federal, a través de la Secretaría de Gobernación, por conducto del Consejo Nacional de Población y el Gobierno del Estado con el objetivo establecer, coordinar y evaluar la aplicación de la política de población en la entidad", en Periódico Oficial del Gobierno del Estado Libre y Soberano de San Luis Potosí, 26 de abril de 2003.

"Acuerdo de Coordinación que celebran, por una parte, el licenciado Patrocinio González Blanco Garrido, secretario de Gobernación, en representación del Ejecutivo federal, y el doctor Manuel Urbina Fuentes, secretario general del Consejo Nacional de Población y, por la otra, el licenciado Horacio Sánchez Unzueta, gobernador constitucional del estado y presidente del Consejo Estatal de Población, así como el licenciado Jaime Suarez Altamirano, secretario general de Gobierno y el licenciado Leonardo Hernández Covarrubias, secretario técnico del Consejo Estatal de Población”, en Periódico Oficial del Gobierno del Estado Libre y Soberano de San Luis Potosí, 8 de febrero de 1994.

Consejo Estatal de Población, Informe anual de actividades de gobierno, 20142015, p. 7, mecanuscrito.

Consejo Internacional del Archivos, ISAAR (CPF): Norma Internacional Sobre los Registros de Autoridad de Archivos relativos a Instituciones, Personas y Familias, traducción española de ISAAR (CPF). International Standard Archival Autoritby Records for Corporate Bodies, Persons and Families, 2a ed., Madrid, Subdirección de los Archivos Estatales, 2004.

Consejo Internacional del Archivos, ISAD (G): Norma Internacional General de Descripción Archivistica: adaptada por el Comité de Normas de Descripción, Estocolmo, Suecia, 19-22 de septiembre 1999, versión española de Asunción de Navascués Benlloch, 2a ed., Madrid, Subdirección de los Archivos Estatales, 2000.

Cruz Mundet, José Ramón (dir.), Administración de documentos y archivos: textos fundamentales, Madrid, España, Coordinadora de Asociaciones de Archiveros, 2011. 